International Journal of Engineering \& Technology, $7(3.12)(2018) 1161-1167$
International Journal of Engineering \& Technology
SPC
Website: www.sciencepubco.com/index.php/IJET
Research paper

\title{
Experimental Study and Parameter Optimization of Hybrid Electrical Discharge Machining
}

\author{
Nishant Kumar Singh ${ }^{1 *}$, Sandeep Agrawal', Rajvardhan $^{3}$, Yashvir Singh ${ }^{4}$ \\ ${ }^{1,2,3}$ Department of Mechanical Engineering, Hindustan College of Science \& Technology, Mathura \\ ${ }^{4}$ Department of Mechanical Engineering, University of Petroleum and Energy Studies, Dehradun
}

\begin{abstract}
Hard materials cannot be machined effectively by the individual machining process. In order to machine workpiece made from hard and stiff materials effectively a concept of Hybrid machining process (HMP) is originated. The HMP is an integration of two or more machining process to get the advantage of each individual process. HMP is used to machine This study focuses on evolving a novel process using both oil and gas as dielectrics to analyse the effect on tool wear rate (TWR) and material removal rate (MRR). The flow of compressed gas through eccentric-hole rotating tool improved the debris removal from inter-electrode gap, hence it improve the flushing competence of the machining process. In this experimental investigation, the workpiece material is $\mathrm{Al}-20 \% \mathrm{SiC}$ metal matrix composite (MMC) and the electrode material is copper. The experiments were conducted following the Taguchi method of design experiments. The effect of various machining parameters on MRR and TWR has been studied. The optimization of process parameter has also been done. The results of TWR and MRR are analysed using S/N ratio, ANOVA and main effect plots. The experimental results, revels that discharge current, gap voltage and pulse on time significantly affected MRR, and TWR. The experimental inference reveal that provision of compressed air through eccentric hole rotary tool has a positive effect on machinability of electrical discharge machining (EDM) process.
\end{abstract}

Keywords: EDM, MRR, TWR, Taguchi Method, ANOVA, S/N ratio, Optimization.

\section{Introduction}

Electrical Discharge Machining (EDM) is a most widely used method to fabricate molds, dies, aerospace and automotive components [1]. Surface finish of machined specimens in EDM process is affected by many process factors. From literature it has been observed that apart from machining parameters, electrical and thermo-mechanical properties of electrodes are the factors that significantly affect the surface finish of workpiece machined through EDM process. The most commonly used dielectric fluid is kerosene [2]. The tool is generally made of a conducting material such as brass or copper.

\section{Principle of EDM in gas}

Dielectric liquid is essential during machining process as it imparts invariability and improves efficacy of the process. It helps as the cooling medium and flushes out the wear particles produced during machining. Thus, it plays a vital role during material removal mechanism. During machining, harmful vapors $(\mathrm{CO}$ and $\mathrm{CH}_{4}$ ) are released that could harm the health of the machine operator. Nowadays, sustainable method of EDM has turn out to be an important subject of the study around the globe.

Kunieda and Yoshida [3] proposed new EDM machining method using a gas as a dielectric medium. In this method, gas based dielectrics have been used which results in the reduction of pollution. Numerous literatures are available based on gas assisted EDM studies which also describes about the process involved. [5-
8]. The effect of various factors on the MRR has also been studied $[9,10]$.

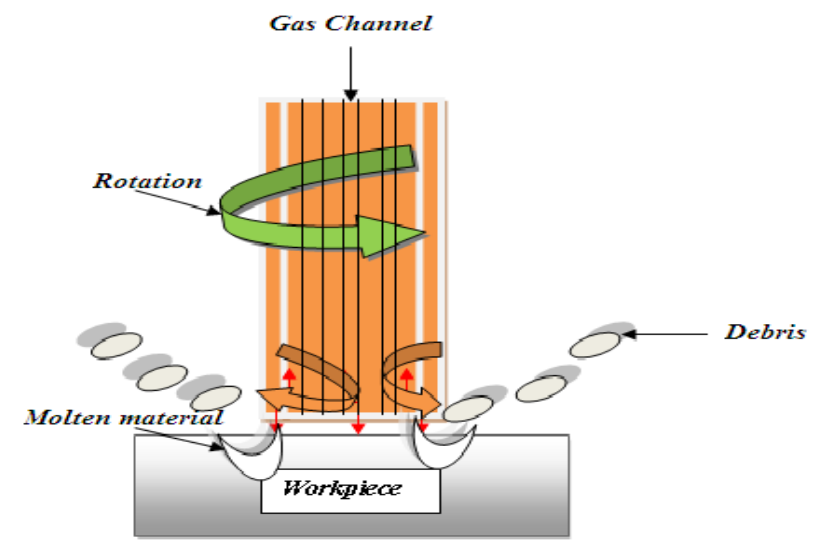

Fig.1: Principle of EDM in gas [2]

Figure 1 shows the principle of EDM in gas. High speed gas assisted jet removes the molten material from the tool and workpiece. The pressurized gas helps in the removal of the material which is further gets solidifies after getting cool. This process prevents materials from getting adhere to the surface of tool electrode and the machined specimen. The greatest ease of $\mathrm{EDM}$ in gas medium is that the tool wear is very less irrespective of machining conditions. Various un-conventional approaches have also been used with conventional EDM to enhance its functioning. Mohan et al. [11] investigated the electrical discharge machining of ceramic metal matrix composite (MMC) and 
analysed the effect of tool speed on the machinability of process. Kuppan et al. [12] analysed the influence of tool rotation during EDM drilling of Inconel 718.

Teimouri and Baseri [13] investigated the impelled action of electrode rpm and different scale of magnetic field on EDM machinablity. Abdulkareem et al. [14] performed the experimentation to know the effect of cryogenic cooled electrode on process responses during EDM of titanium alloy workpiece. Srivastava and Pandey [15] used ultrasonic assisted cryogenically cooled electrode to evaluate the EDM performance. Authors compared the effectiveness of ultrasonic assisted cryogenically cooled electrode over the conventional electrode and cryogenically cooled electrode in terms of process responses. Aliakbari and Baseri [16] applied the Taguchi based DOE to obtain the optimum process factors for rotary assisted multi hole electrode EDM process and studied the effect of machining factors on responses such as SR, MRR, EWR, and over-cut. Gu et al. [17] performed the feasibility analysis of EDM of Ti6Al4V by using bunched electrode. They performed a comparative analysis on the use of purposed electrode and traditionally used electrode on EDM process responses. Singh and Pandey [17] studied the effect of air assisted multi-hole tool electrodes during machinability of EDM process. They found the application of air assisted multihole tool improve the MRR and reduced the EWR with respect to solid rotary tool electrodes under same machining conditions. Yoshida et al [18] studied the effect of supplied of oxygen gas in dielectric liquid in EDM operation. They observed that when oxygen is dissolved in dielectric liquid and supplied in electrodes gap machining performance improve significantly.

Hybrid machining process (HMP) is integration of two or more machining process. HMP is used to machine hard materials that cannot be machined effectively by individual machining process. HMP is generally used to take the benefits of the each individual process so that machinability can be enhanced. The present research work, emphasis on evolving a process using both liquid and gas as dielectrics, to extricate the advantage of gas based EDM and conventional EDM, during machining of Al-SiC metal matrix composite (MMC) with rotary eccentric hole tool during die-sinking EDM operation. A comparative study has been performed to compare MRR and TWR of gas assisted hybrid EDM and oil EDM process. The objective of present study was experimental investigation and optimization of MRR and TWR by using a gas assisted EDM process.

\section{Experimental Details}

\subsection{Work Piece Details}

Fig.2. shows the EDX paradigm of $\mathrm{Al}-20 \% \mathrm{SiC}_{\mathrm{p}} \mathrm{MMC}$. It is apparent from Table1 that $\mathrm{Al}, \mathrm{Si}, \mathrm{C}, \mathrm{O}, \mathrm{Ni}$ are the key constituents in the re-solidified layers.

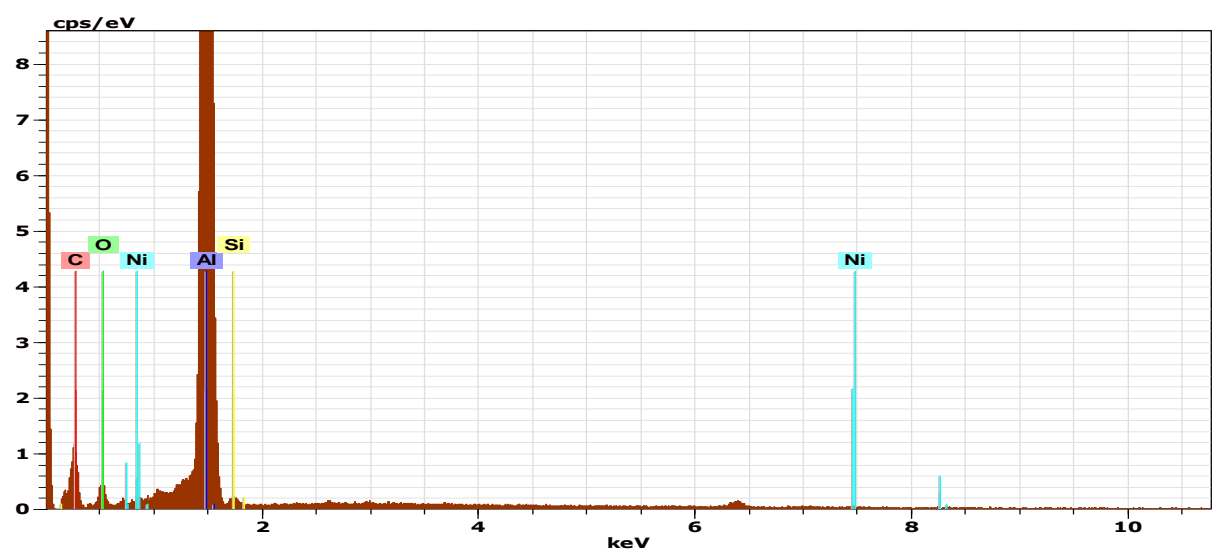

Fig. 2: $\mathrm{EDX}$ of $80 \% \mathrm{Al} \& 20 \%$ Sic MMC

Table 1: Characterization of Work piece

\begin{tabular}{|c|c|c|c|c|c|}
\hline Element & $\mathrm{Al}$ & $\mathrm{C}$ & $\mathrm{Si}$ & $\mathrm{Ni}$ & Others \\
\hline$\%$ & 79.34 & 12.33 & 5.26 & 1.89 & 1.18 \\
\hline
\end{tabular}

\subsection{Experimental Design}

The Taguchi design of experiments is used to analyze the influence of input parameters on responses. The effectiveness of design of experiment depends upon the selection of the process parameters [23]. From review of previous literatures it was observed that parameters that have a notable influence on the EDM process performance was discharge current, pulse-on time, duty cycle, and gap voltage. Therefore, these four parameters are decided for the present experimentation. As per Taguchi design of experiments total of nine experiments were carried out with independent variables at three different levels. On the basis of exploratory experimentation performed and capability of EDM machine, the levels of the discharge current, pulse-on time, duty cycle, and gap voltage were selected as 3-7 A, 100-500 $\mu$ s, duty cycle $0.40-0.70$, and $50-70 \mathrm{~V}$, respectively $[19,22]$. In hybrid and conventional EDM experimentation the rpm of the tool was kept constant at $500 \mathrm{rpm}$ while, the air was supplied at $12 \mathrm{~mm} \mathrm{Hg}$ to the inter electrodes gap through three eccentric hole rotary tool electrode during die-sinking EDM operation.

Machining time for each experiment was fixed to 10 minute. The levels of process factors are depicted in Table 2.
Table 2: Machine factors with levels

\begin{tabular}{|c|c|c|c|c|c|}
\hline S.No & Parameter & Unit & Level1 & Level2 & Level3 \\
\hline 1 & Current & $\mathrm{A}$ & 3 & 5 & 7 \\
\hline 2 & Pulse on time & $\mu \mathrm{s}$ & 100 & 300 & 500 \\
\hline 3 & Duty Cycle & & 0.40 & 0.56 & 0.70 \\
\hline 4 & Voltage & $\mathrm{V}$ & 50 & 60 & 70 \\
\hline
\end{tabular}

\subsection{Experimental Setup}

The experimentations were performed on EDM machine (Model ZNC, Electronica, India). The experimental setup used for present experimental work is shown in figure 3. Eccentric three hole tool having outer and inner diameters of $10 \mathrm{~mm}$ and $8 \mathrm{~mm}$, respectively was used during the experimentations. Figure 4 show the view of tool used during the experimentation. Belt-pulley arrangement is used to rotate spindle shaft and continuous speed control of the motor is possible through rheostat control system [25]. Air compressor which has a built-in air drier is used to obtain high pressure air 


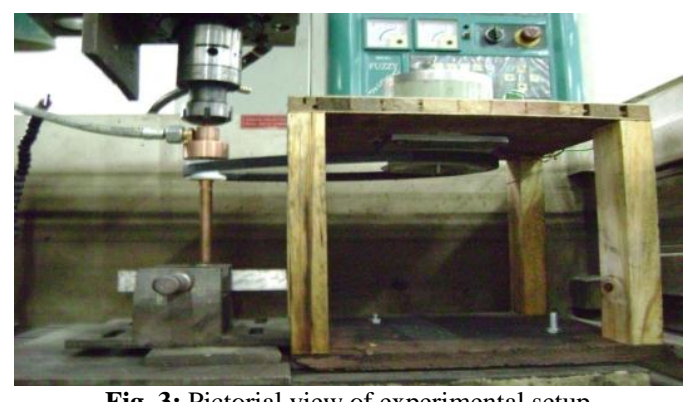

Fig. 3: Pictorial view of experimental setup

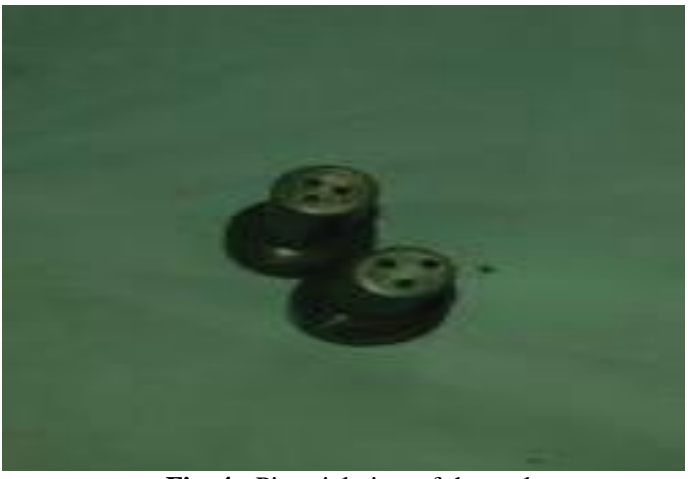

Fig. 4: Pictorial view of the tool

\subsection{Measurement of MRR and TWR.}

The machined specimens were cleaned with acetone after every experiment and weight loss of electrodes was evaluated by machine having least count $0.1 \mathrm{mg}$.

a) TWR (g/min)

Tool wear rate is the ratio of the material extracted from the tool to the machining time [24]. It is expressed in $\mathrm{gm} / \mathrm{min}$.

$$
T W R=\frac{\text { Material removed from the tool }}{\text { Machining time }}
$$

Here, weight loss is expressed in grams.

b) $\quad M R R=\frac{\text { Material removal from specimen }}{\text { Machining Time }}$

\section{Results and Discussion}

\subsection{The Experimental Condition:}

Table 2: Experimental results with kerosene only

\begin{tabular}{|c|c|c|}
\hline $\begin{array}{c}\text { Exp. } \\
\text { No. }\end{array}$ & $\begin{array}{c}\text { MRR } \\
(\mathrm{g} / \mathrm{min})\end{array}$ & $\begin{array}{c}\text { TWR } \\
(\mathrm{g} / \mathrm{min})\end{array}$ \\
\hline 1 & 0.0277 & 0.00136 \\
\hline 2 & 0.0208 & 0.00130 \\
\hline 3 & 0.0181 & 0.00128 \\
\hline 4 & 0.0204 & 0.00131 \\
\hline 5 & 0.0198 & 0.00127 \\
\hline 6 & 0.0398 & 0.00135 \\
\hline 7 & 0.0171 & 0.00125 \\
\hline 8 & 0.0382 & 0.00134 \\
\hline 9 & 0.0284 & 0.00130 \\
\hline
\end{tabular}

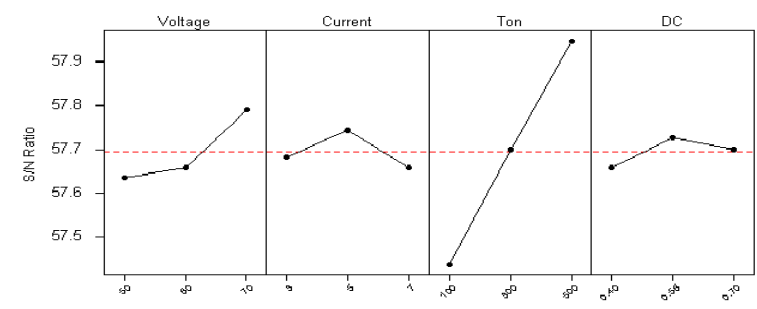

Fig.5 (a): Effect of S/N ratio on tool wear
Table 3: Experimental results with kerosene and air

\begin{tabular}{|c|c|c|}
\hline $\begin{array}{c}\text { Exp. } \\
\text { No. }\end{array}$ & $\begin{array}{c}\text { MRR } \\
(\mathrm{g} / \mathrm{min})\end{array}$ & $\begin{array}{c}\text { TWR } \\
\left(\mathrm{g}^{\prime} \text { min }\right)\end{array}$ \\
\hline 1 & 0.0277 & 0.00105 \\
\hline 2 & 0.0208 & 0.00102 \\
\hline 3 & 0.0181 & 0.00103 \\
\hline 4 & 0.0204 & 0.00101 \\
\hline 5 & 0.0198 & 0.00104 \\
\hline 6 & 0.0398 & 0.00113 \\
\hline 7 & 0.0171 & 0.00113 \\
\hline 8 & 0.0382 & 0.00108 \\
\hline 9 & 0.0284 & 0.00111 \\
\hline
\end{tabular}

Table 4: Reduction in tool wear by using air

\begin{tabular}{|c|c|c|c|}
\hline $\begin{array}{c}\text { Exp. } \\
\text { No. }\end{array}$ & $\begin{array}{c}\text { \% TWR With } \\
\text { Kerosene only }\end{array}$ & $\begin{array}{c}\text { \% TWR With } \\
\text { Kerosene \& air }\end{array}$ & $\begin{array}{c}\text { Reduction in } \\
\text { tool wear } \\
(\%)\end{array}$ \\
\hline 1 & 0.0491 & 0.0379 & 22.81 \\
\hline 2 & 0.0625 & 0.0490 & 21.6 \\
\hline 3 & 0.0707 & 0.0569 & 19.51 \\
\hline 4 & 0.0642 & 0.0495 & 22.89 \\
\hline 5 & 0.0641 & 0.0525 & 18.09 \\
\hline 6 & 0.0339 & 0.0284 & 16.22 \\
\hline 7 & 0.0735 & 0.0605 & 17.68 \\
\hline 8 & 0.0350 & 0.0282 & 19.42 \\
\hline 9 & 0.0457 & 0.0390 & 14.66 \\
\hline
\end{tabular}

Table 5: Increase in MRR by using air

\begin{tabular}{|c|c|c|c|}
\hline $\begin{array}{c}\text { Experiment } \\
\text { No }\end{array}$ & $\begin{array}{c}\text { MRR With } \\
\text { Kerosene only }\end{array}$ & $\begin{array}{c}\text { MRR With } \\
\text { Kerosene \& air }\end{array}$ & $\begin{array}{c}\text { Increase in } \\
\text { MRR (\%) }\end{array}$ \\
\hline 1 & 0.0277 & 0.0332 & 19.85 \\
\hline 2 & 0.0208 & 0.0254 & 22.11 \\
\hline 3 & 0.0181 & 0.0224 & 23.75 \\
\hline 4 & 0.0204 & 0.025 & 22.54 \\
\hline 5 & 0.0198 & 0.0237 & 19.69 \\
\hline 6 & 0.0398 & 0.0472 & 18.59 \\
\hline 7 & 0.017 & 0.0211 & 24.11 \\
\hline 8 & 0.0382 & 0.0462 & 20.94 \\
\hline 9 & 0.0284 & 0.0347 & 22.18 \\
\hline
\end{tabular}

From table 3,4 , and 5, it can be inferred that use of air results in increase in MRR, reduction in TWR and also reduction in percentage electrode wear. Table 4 gives the percentage reduction in tool wear with the use of compressed air. Maximum reduction of tool wear is found in experiment 4 which is $22.89 \%$ and maximum increase in MRR is $24.11 \%$.

\subsection{Main Effects}

\subsubsection{Main Effects for TWR with Kerosene}

From main effects plots as shown in figure 5(a) and 5(b), it was observed that the tool wears increases as the voltage was increased from $50 \mathrm{~V}$ to $60 \mathrm{~V}$ and then decreases when voltage increased from $60 \mathrm{~V}$ to $70 \mathrm{~V}$. The tool wears increases when discharge current was increased. It was probably due to higher discharge energy available in machining gap [19]. Further it was observed tool wears increases with enhancement in pulse on time and duty cycle. This may be because of the fact that, the energy is supplied for longer pulse duration, which increases the temperature near the electrode tip [20, 26]. 


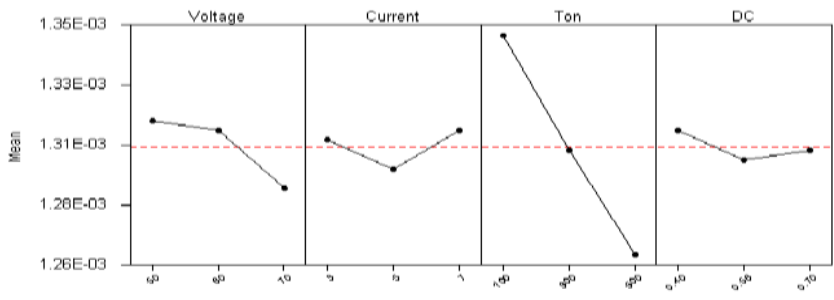

Fig. 5 (b): Effect of process parameter on tool wear

4.2.2 Main Effects for TWR with Kerosene and Air
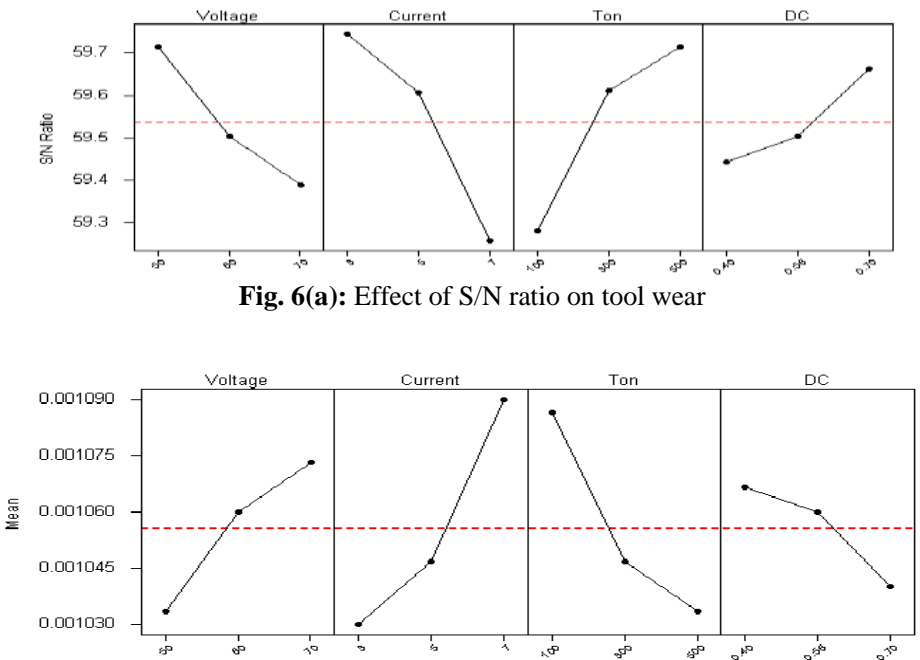

Fig. 6(b): Effect of process parameter on tool wear

From main effects plots as shown in figure 6(a) and 6(b) of tool wear, it has been seen that tool wear increases with increased in voltage, pulse on time and duty cycle. This was likely due to reason that high current generated more discharge energy. This results in elevated temperature in the machining region because of which electrode wear increased along with MRR [21]. Further, from plot it has also been revealed that tool wear increases with increase in current and then decreases when current increases from $5 \mathrm{~A}$ to $7 \mathrm{~A}$

\subsubsection{Main Effects for MRR with Kerosene}

From main effects plots as shown in figure 7(a) and 7(b), of MRR, it has been seen that MRR get enhanced with increasing the gap voltage as well as discharge current. This is probably due to an increase in discharge energy and enlargement of dimensions craters at the discharge point $[20,21]$.

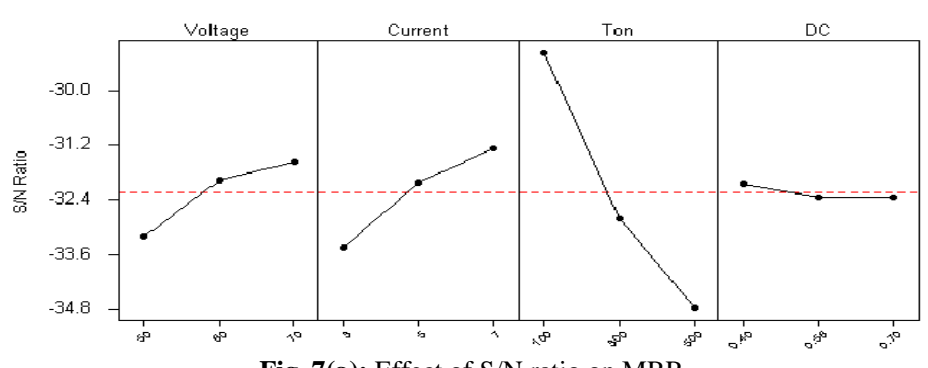

Fig. 7(a): Effect of $\mathrm{S} / \mathrm{N}$ ratio on $\mathrm{MRR}$

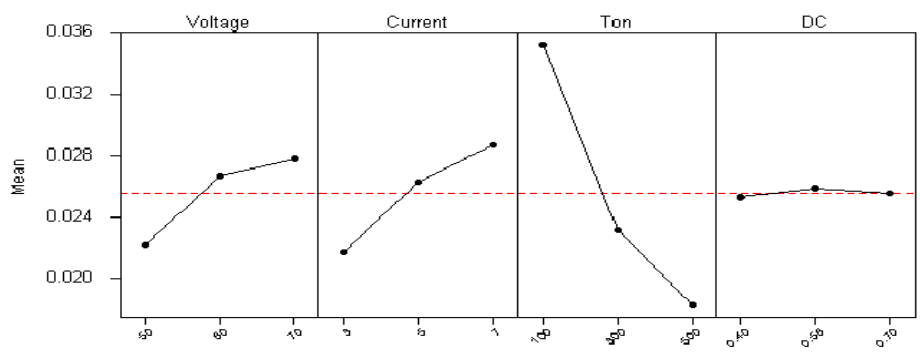

Fig. 7(b): Effect of process parameter on MRR 
Further from the plot it was revealed that MRR decreased with increase in pulse on time. It was probably due to the reason that in rotary EDM, long pulse duration led to plasma channel expansion, so the energy density decreased. Therefore, less melting and vaporization of workpiece resulted in a reduction of MRR at longer pulse duration [25]. Further as evident from figure it can be noticed that MRR increased with an enhancement in duty cycle. It was likely because of the reason that the increase of duty cycle means applying the higher spark discharge energy and this caused an enlarged MRR [25]

\subsubsection{Main Effects for MRR with Kerosene and Air}

From main effects plots as shown in figure 8(a) and 8(b), of MRR, with hybrid EDM process, similar trends had observed as it observed with oil based EDM process. The MRR was found to be more when hybrid EDM process was used as compared with oil based EDM process.

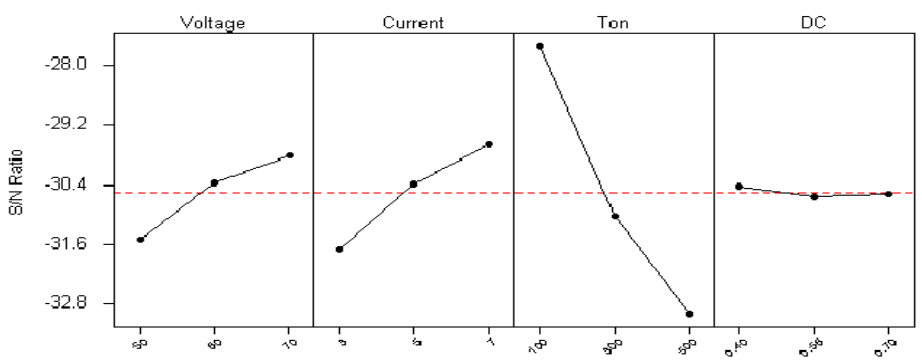

Fig. 8(a): Effect of $\mathrm{S} / \mathrm{N}$ ratio on $\mathrm{MRR}$

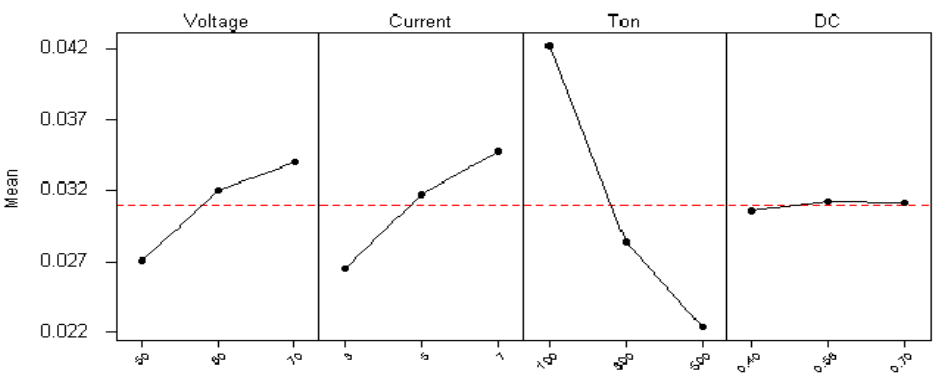

Fig. 8(b): Effect of process parameter on MRR

This was probably due to extra heat that released during exothermic reaction (due to presence of oxygen in air) caused a more of melting and evaporation of workpiece material [5, 6, 17] Further compressed air removed eroded particles from discharge gap without getting attached on the surface of specimen and hence improved the flushing of the process resulting in an enhanced MRR.

\section{Analysis of Variance (ANOVA)}

ANOVA is done to know the parameters that can notably affected the process responses. The optimal value of parameters was determined for both processes on the basis of average $\mathrm{S} / \mathrm{N}$ ratio of each parameter at every level [22]. The level for which $\mathrm{S} / \mathrm{N}$ ratio is largest is the optimal level for that parameter.

It is because larger S/N ratio gives the better quality. Table 6 and 7 represents the optimal value of parameters for MRR and TWR respectively.

Table 6: Optimal value of parameter for MRR

\begin{tabular}{|c|c|c|}
\hline Parameter & With kerosene only & With kerosene and air both \\
\hline Current & $7 \mathrm{~A}$ & $7 \mathrm{~A}$ \\
\hline Pulse on Time & $100 \mu \mathrm{s}$ & $100 \mu \mathrm{s}$ \\
\hline Duty Cycle & 0.70 & 0.70 \\
\hline Gap Voltage & $70 \mathrm{~V}$ & $70 \mathrm{~V}$ \\
\hline
\end{tabular}

Table 7: Optimal value of parameter for TWR

\begin{tabular}{|c|c|c|}
\hline Parameter & With kerosene only & With kerosene and air both \\
\hline Current & $3 \mathrm{~A}$ & $3 \mathrm{~A}$ \\
\hline Pulse on Time & $500 \mu \mathrm{s}$ & $500 \mu \mathrm{s}$ \\
\hline Duty Cycle & 0.40 & 0.40 \\
\hline Gap Voltage & $70 \mathrm{~V}$ & $70 \mathrm{~V}$ \\
\hline
\end{tabular}

\subsection{Percentage Contribution of Various Factors}

The values of square sums (SS) obtained from ANOVA for TWR in case of $80 \% \mathrm{Al} \& 20 \% \mathrm{SiC}$ MMC: With Kerosene only and Kerosene and air. The contribution (\%) of each parameter affecting the TWR and MRR is obtained on the basis of SS-value obtained through ANOVA. On the basis of these analyses pi-chart is plotted for TWR and MRR respectively.

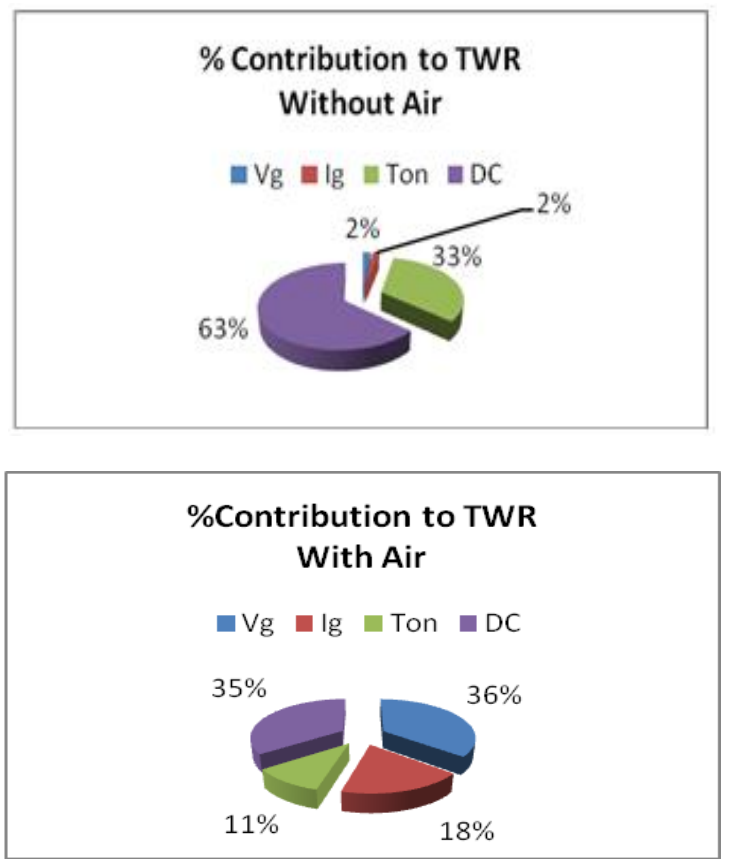

Figure 9: Contribution (\%) of various factors for TWR 
In case of TWR as shown in figure 9, while using kerosene only as dielectrics, duty cycle is most significant factors, and then comes pulse on time while effect of gap voltage and discharge current is insignificant. On employing air as well in the design experiment, contribution of pulse on time and duty cycle is lowers while discharge current and gap voltage increases. It is probably due to cooling and flushing effect provided by the air that pulse on time and duty cycle becomes less significant.
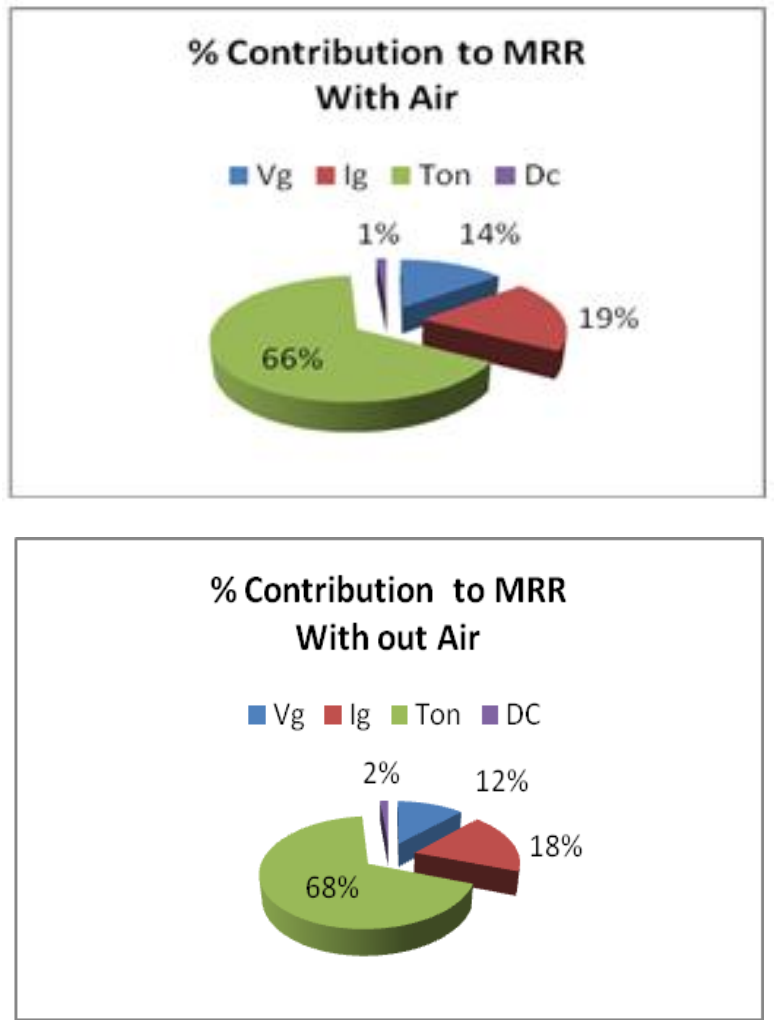

Figure 10: Contribution (\%) of various factors for MRR

In case of MRR as shown in figure 10, while using kerosene only as dielectrics, pulse on time is most significant factors, and then comes discharge current while duty cycle is insignificant. On employing air as well in the design experiment, contribution of pulse on time is lowers while discharge current increases. It is probably due to cooling and the flushing as well as oxidizing effect provided by air that pulse on time becomes less significant and discharge current increases.

\section{Conclusion}

In this study, the effect of a hybrid process as well as influence of machining factors on MRR, and TWR during EDM of Al-20\% $\mathrm{SiC}_{\mathrm{p}} \mathrm{MMC}$ was investigated. The conclusion of this study is as stated below.

- The application of compressed air through rotary eccentric hole tool led to reduction in TWR and improvement of MRR during die-sinking EDM operation.

- In hybrid EDM process in comparison of conventional EDM process TWR are found to be less than $23 \%$. Moreover, MRR found to be more than $25 \%$ in comparison of conventional EDM process.

- The $\mathrm{S} / \mathrm{N}$ ratio based analysis gives the optimal values of the all the parameters for which largest reduction in TWR and maximum MRR can be obtained. The optimal value of parameters for maximum reduction in tool wear was gap voltage $70 \mathrm{~V}$, discharge current $3 \mathrm{~A}$, and pulse on time $500 \mu \mathrm{s}$ and duty cycle 0.40. Likewise, optimal values of parameters for maximum MRR was gap voltage $70 \mathrm{~V}$, discharge current $7 \mathrm{~A}$, and pulse on time $100 \mu$ s and duty cycle 0.70 .
The ANOVA analysis gives the idea about the machining factors which had significant affect on the TWR and MRR. The result reveal that, gap voltage and duty cycle were most prominent factor affecting hybrid EDM process. However, duty cycle was most notable factor followed by pulse on time in traditional EDM process.

- $\quad$ Experimental inference indicated that flow of compressed air through rotary eccentric hole tool during EDM operation have a positive effect on process responses.

\section{References}

[1] K.H. Ho, S.T. Newman, State of the art electrical discharge machining (EDM). Int. J. Mach. Tools Manuf. 43(2003) 1287 300.

[2] N.K. Singh, P.M. Pandey, K.K. Singh, M. K. Sharma, Steps towards green manufacturing through EDM process: A review. Cogent Engineering 2016, 3: 1272662, doi: $10.1080 / 23311916.2016 .1272662$

[3] M. Kuineda, S. Furuoya, Improvement of EDM Efficiency by Supplying Oxygen Gas into Gap. CIRP Annals- Manuf. Technol. 40(1991), 215-218.

[4] M. Kunieda, M. Yoshida, Electrical discharge machining in gas Annals of CIRP, 46 (1997), 143-146.

[5] Q.H. Zhang, J.H. Zhang, J.X. Deng, Z.W. Niu, Ultrasonic vibration in electrical discharge machining. J. Mater. Process Tech. 129(2002), 135-138

[6] Q.H. Zhang, J.H. Zhang, J.X. Deng, S.F. Ren, Study on technology of ultrasonic vibration aided electrical discharge machining in gas. J. Mater. Process Tech. 149(2004), 640-644.

[7] Q.H. Zhang, J.H. Zhang, Q.B. Zhang, An Investigation of ultrasonic-assisted electrical discharge machining in gas. Int. $\mathrm{J}$ Mach. Tools Manuf. 46 (2005), 1582-1588.

[8] J. Tao, A.J. Shih, J. Ni, Experimental study of the dry and near dry electrical discharge milling process. J. Manuf. Sci. Eng. 130 (1) (2008), 1-8.

[9] P. Govindan, S.S. Joshi, Investigation into Performance of dry EDM using slotted electrodes. International J. Precis. Eng. Manuf. 12(6) (2011), 957-963

[10] R. Roth, F. Kuster, K. Wenger, Influence of Oxidizing Gas on the Stability of Dry Electrical Discharge Machining Process. Procedia CIRP, 6(2013), 339-344.

[11] B. Mohan, A. Rajadurai, K.G. Satyanarayana, Effect of SiC and rotation of electrode on electric discharge machining of $\mathrm{Al}-\mathrm{SiC}$ composite. J. Mater. Process Tech. 124(2002), 297-3042.

[12] P. Kuppan, A. Rajadurai, S. Narayanan, Influence of EDM process parameters in deep hole drilling of Inconel 718. Int. J. Adv. Manuf. Tech. 38(2008), 74-84.

[13] Teimouri R, Baseri H. Effects of magnetic field and rotary tool on EDM performance. J Manuf. Process 2012; doi: 10.1016/j. jmapro.2012.04.002

[14] AbdualKareem S, Khan A.A, Konneh M. Reducing electrode wear ratio using cryogenic cooling during electrical discharge marching. Int J Adv Manuf Tech 2009; 45: 1146-1151.

[15] Srivastava V, Pandey PM. Effect of process parameters on the performances of EDM process with ultrasonic assisted cryogenically cooled electrode. J Manuf Process 2012; 14: 393-402.

[16] E. Aliakabari, H. Baseri, Optimization of machining parameters in rotary EDM process by using the Taguchi method. Int. J. Adv. Manuf. Tech. 62 (9) (2012), 1041-1053.

[17] Singh NK, Pandey PM, Singh KK (2016) EDM with Air Assisted Multi-Hole Rotating Tool. Mater Manufac Processes 31 (14): 1872 1878.

[18] Yoshida M, Ishii Y, Ueda T (2017) Study on electrical discharge machining for cemented carbide with non-flammable dielectric liquid: Influence of form of oxygen supplied to dielectric liquid on machining Proc IMechE Part B: J Engg Manufac, DOI: $10.1177 / 0954405417706995$

[19] Gu L, Li L, Zhao W. Electrical discharge machining of Ti6Al4V with a bundled electrode. Int J Mach Tools Manuf 2012; 53:100 106.

[20] A. Singh, P. Kumar, I. Singh, Electrical discharge drilling of metal matrix composites with different tool geometries. J. Process Mech. Engg. 2013; doi: 10.1177/0954405413484726.

[21] K.D. Chattopadhyaya, S. Verma, P.C. Satsangi, P.C. Sharma, Development of empirical model for different process parameters 
during rotary electrical discharge machining of copper-steel (EN-8) system. J. Mater. Proc. Tech. 209 (3) (2009), 1454-1465.

[22] R. Karthikeyan, L. Narayanan, R.S. Naagarazan, Mathematical modeling for electric discharge machining of $\mathrm{Al}-\mathrm{SiC}$ particulate composites. J. Mater. Process. Tech. 87(1999), 59-63.

[23] K.M. Patel, P.M. Pandey, V. P. Rao, Study on Machinability of $\mathrm{Al} 2 \mathrm{O} 3$ ceramic composite in EDM using Response Surface Methodology. J. Eng. Mater. Tech. 133(2011), 021004-9.

[24] R Teimouri, H. Baseri, Effects of magnetic field and rotary tool on EDM performance. J. Manuf. Process 2012, doi:10.1016/j.jmapro.2012.04.002.

[25] N.K. Singh, P.M. Pandey, K.K. Singh, Experimental investigations into the performance of EDM using argon gas-assisted perforated electrodes Materials and Manufacturing Processes 2016; doi: 10.1080/10426914.2016.1221079

[26] V. Srivastava, P.M. Pandey, Effect of process parameters on the performances of EDM process with ultrasonic assisted cryogenically cooled electrode. J. Manuf. Process. 14 (3) (2012), 393-402. 